\title{
MODELO DE GESTIÓN PARA EL SUMINISTRO DE MATERIALES E INSUMOS BASADO EN LA DEMANDA
}

\author{
Isabel Cristina Arango Palacio \\ Empresa de Servicios y Cosméticos SEMCO S.A. \\ proveedores@semcocosmetics.com
}

\author{
Abdul Zuluaga Mazo \\ Politécnico Colombiano Jaime Isaza Cadavid \\ azuluaga@elpoli.edu.co
}

(Tipo de Artículo: Investigación. Recibido el 18/11/2014. Aprobado el 09/12/2014)

\begin{abstract}
RESUMEN.
Este artículo hace referencia a la gestión de la cadena de abastecimiento, la cual requiere la sincronización de actividades que incluyen el flujo de información e inventarios. Una gestión exitosa depende de la óptima integración y sincronización de las actividades e inventarios requeridos para satisfacer la demanda. A partir de la investigación realizada en una empresa de cosméticos de venta directa, se analizan las estrategias para mejorar la gestión del suministro enfocado a la demanda, de modo que se pueda responder al mercado y, al mismo tiempo, controlar los inventarios y evitar los agotados.

En esta investigación se toman en consideración que el suministro basado en el consumo diario, con entregas frecuentes y lotes pequeños flexibiliza el abastecimiento con los cambios de la demanda, y el stock de seguridad en cada empresa de la cadena de abastecimiento se convierte en un amortiguador para proteger la variabilidad. La metodología comienza con la identificación del problema, las posibles causas y las relaciones con los efectos encontrados con el fin de obtener un modelo de gestión del abastecimiento basado en la demanda, para responder rápidamente a la sobredemanda sin generar agotados y, a la vez, reaccionar a la subdemanda sin exceso de inventario.
\end{abstract}

Palabras clave. Cadena de abastecimiento, Pronósticos, Reposición, Inventarios, Flujo de productos.

\section{MANAGEMENT MODEL BASED ON THE DEMAND FOR THE PROVISION OF MATERIALS AND SUPPLIES}

\begin{abstract}
.
This article deals with managing the supply chain, which requires synchronizing the activities that include information flow and inventory. A successful management depends on the optimal integration and synchronization of the activities and required inventories in order to satisfy the demand. Based on the research conducted in a direct-selling beauty product enterprise we analyze the strategies to improve the management of supply focused on the demand, in such a way that make possible meet the market and, at the same time, controlling the inventories and avoiding goods out of stock.

This research considers that the supply based on daily consumption, with frequent delivers and small batches, increases the flexibility of the supply related to demand changes, and the security stock in every enterprise of the supply chain becomes a damper in order to protect the variability. Thus, it proposes a management model of supply based on demand in order to quickly meet overdemand without producing goods out of stock, and at the same time, reacting to underdemand without inventory excess.
\end{abstract}

Keywords. Supply Chain, Forecast, Replenishing, Inventories, Product Flow.

\section{Modèle de gestion pour l'approvisionnement de matériels et intrants en se basant sur la demande}

\section{Résumé.}

Cet article fait allusion à la gestion de la chaine d'approvisionnement, cette gestion demande la synchronisation des activités en incluant le flux d'information et des inventaires. Une gestion à succès dépend de l'optimale intégration et synchronisations des activités et des inventaires requis afin de satisfaire la demande. À partir de la recherche réalisée dans un entreprise de produits cosmétiques de vente directe, on analyse les stratégies pour améliorer la gestion de l'approvisionnement dirigé vers la demande, de sorte que on peut répond au marché, et au même temps, de régler les inventaires et éviter épuiser les stocks.

Dans cette recherche on considère que l'approvisionnement qui se base sur la consommation quotidien, avec des remises fréquents et lots petits, flexibilise l'approvisionnement avec les changes de la demande et le stock de sécurité dans chaque entreprise de la chaine d'approvisionnement devient dans un amortisseur pour protéger la variabilité. Par conséquent, on propose un modèle de gestion de l'approvisionnement qui se base sur la demande pour répondre rapidement à la sur-demande sans produire stocks épuisés et, en même temps, de répondre a la sous-demande sans excès d'inventaire.

Mots-clés. Chaine d'approvisionnement, Pronostics, Reposition, Inventaires, Flux des produits 


\section{INTRODUCCIÓN}

Cuando un cliente hace un pedido, no significa que se tiene disponibilidad inmediata para su despacho; de modo que es preciso sincronizar diferentes actividades para garantizar la disponibilidad de materiales e insumos, producir en forma ágil y distribuir de manera oportuna el producto hasta el consumidor final. En este caso la logística integral de la cadena de abastecimiento es fundamental para garantizar que estas actividades se realicen de forma rápida y eficiente.

La Empresa de Cosméticos S.A. cuenta con más de 200 trabajadores, ventas anuales aproximadas de $\$ 12.000$ millones de pesos. Clasificada como mediana empresa según la ley 590 del 2004, la cual reglamenta la promoción y desarrollo de la micro, pequeña y mediana empresa [1]. Ofrece productos terminados de marca propia y maquila para productos solicitados por clientes con diferentes marcas. Cuenta dentro de su infraestructura con un laboratorio especializado para controlar las características microbiológicas requeridas por el INVIMA para productos de consumo humano, cuenta con máquinas pesadoras, mezcladoras, envasadoras y empacadoras, para transformar los materiales en producto terminado.

En la Empresa de Cosméticos S.A. donde se realizó la investigación, el mercado genera pedidos reales en un $300 \%$ adicional a lo pronosticado y en la subdemanda los pedidos reales pueden ser solo un $20 \%$ de lo presupuestado. ¿Cuánto inventario se debería tener para atender la alta variabilidad del mercado y en dónde tener dicho inventario?

El problema que amerita el estudio, está evidenciado en el crecimiento de los inventarios (incremento cerca del $70 \%$ desde el 2008), la poca rotación de los mismos (algunos materiales, cuyo consumo mínimo por parte del proveedor es de rotación lleva a una rotación de 2 veces en el año) lo cual pone en riesgo la liquidez y la alta exigencia del mercado por cumplir los pedidos en el tiempo acordado (dada la alta competencia de empresas cosméticas en publicidad y promociones agresivas). Las decisiones que se deben tomar son rápidas, dejando poco tiempo para analizar su impacto, esto puede poner en peligro el control de los inventarios de materiales.

En la sobredemanda, exige realizar una gestión para el suministro rápido de los proveedores, donde requiere que el proveedor esté disponible para atender solo las urgencias de la empresa, y esto es poco probable. En la subdemanda cuando ya se tiene el inventario disponible, este queda sin rotación hasta el próximo lanzamiento de campaña u ofrecimiento a otros clientes o cuando es posible, se cancelan las órdenes de compra que aún están en el proveedor, generando exceso de inventario, ya sea en la Empresa de
Cosméticos S.A. como en sus proveedores.

Debido a esta situación, la empresa requiere un modelo en la gestión de suministro que permita cumplir el nivel de servicio cuidando la rotación de los inventarios en todas las empresas que participan en la cadena de abastecimiento. La información oportuna entre la cadena de abastecimiento se convierte en una necesidad vital para este propósito.

El aporte de la investigación es diseñar y proponer nuevas estrategias para identificar rápidamente las acciones necesarias con el fin de proteger el nivel de servicio (unidades entregadas a tiempo/unidades solicitadas), depender poco de los pronósticos, planear la demanda a corto plazo (debido al corto tiempo de cada campaña), e integrar los inventarios en la red, a través de su cadena de abastecimiento, y así lograr una rápida reposición de los productos, de acuerdo a la demanda, sin incurrir en excesos de inventario.

El control de los inventarios, la planeación estratégica y la modelación de sistemas dinámicos, son temas investigados para abordar problemas como los excesos y faltantes de materiales en una empresa, en el presente proyecto, se usaran los modelos de simulación con herramienta para estudiar diferentes escenarios, combinada con estrategias de planeación de la producción adaptada a la realidad de la empresa estudiada.

La metodología a seguir, para el desarrollo del presente artículo, es un compendio de un proyecto de investigación específico para la Empresa de Cosméticos S.A., pero que puede ser útil para empresas similares del sector cosmético. Identifica la situación problemática, usa herramientas de modelos de simulación, usa teorías de administración de inventarios conocidas pero adaptadas a la empresa y desarrolla un modelo de gestión de abastecimiento requerido para reponer las necesidades de productos cosméticos para venta directa.

En conclusión podemos identificar cuanto y en donde debe estar el inventario de acuerdo al comportamiento de la demanda, en productos solicitados por el mercado en forma intermitente (intermitente: productos con demandas interrumpidas), teniendo mayor rapidez en la información a través de la cadena de abastecimiento y una adecuada gestión de la red integrada de inventarios.

\section{CONTEXTUALIZACIÓN TEÓRICA}

Entre los temas de investigación que abordan la gestión de las cadenas de abastecimiento, se encuentran la precisión de los pronósticos, la gestión de los inventarios y la gestión de proveedores. Los pronósticos entendidos como los métodos usados para predecir la tendencia de los negocios y realizar mejores planes [2], los inventarios analizados de 
acuerdo a la demanda y los tiempos de reposición, usados para proteger el proceso de toda la cadena de abastecimiento para evitar los paros y excesos [3], y la gestión de los proveedores, entendida como la flexibilidad requerida para responder adecuadamente a la demanda [4].

Para atender la demanda se cuenta con los actores de la cadena de abastecimiento donde se identifica a los proveedores de insumos y material de empaque; ya sean fabricantes o distribuidores, las empresas de fabricación encargadas de convertir los insumos en productos terminados; entre estas se encuentra la Empresa de Cosméticos S.A., otro actor importante son los distribuidores de producto terminado; como grandes cadenas de distribución, retails; en español (venta al detalle), es un sector económico que engloba las empresas especializadas en la comercialización masiva de productos terminados al consumidor final [5], empresas de mercadeo directo; y por último pero no menos importante es el consumidor final; quien dicta las necesidades para reponer a la cadena de abastecimiento en conjunto.

Una de las dificultades que viven las medianas empresas de fabricación de cosméticos, es el desfase tecnológico en maquinaria y equipo, comparado con las grandes empresas de capital extranjero que han aumentado su participación en Colombia, lo que hace que las medianas empresas estén enfocando su mercado a sectores de la población con menores ingresos.

Esta dificultad para acceder a nuevas tecnologías, adicionando el bajo nivel de gestión y el bajo poder negociación con los proveedores de la cadena de abastecimiento, afecta la competitividad de las medianas empresas de cosméticos nacionales [6].

Las dificultades tecnológicas y la variación en la demanda, genera inconvenientes para hacer un buen pronóstico de los SKUS que se comercializan por medio de catálogos, ya que los pedidos son intermitentes, no todos los SKUS se ofertan en cada campaña. La oferta intermitente genera dificultad para seleccionar el mejor modelo de pronóstico. "Un factor importante que influye en la selección de la técnica de elaboración del pronóstico es la identificación de patrones históricos de los datos". John E. Hanke en 2010 recuerda como los patrones históricos ayudan a identificar patrones de tendencia, cíclicos 0 estacionales, para identificar qué modelo de pronóstico usar [2].

Con la dificultad para calcular un buen pronóstico para la demanda, una forma de mejorar la gestión, según algunos autores, es por medio de la implementación de logística integral en la cadena de abastecimiento, la logística integral es conocida como la gestión de la red de proveedores, fabricas, distribuidores y clientes, a través de los cuales fluyen productos o servicios. La referencia [7] muestra en dicha investigación como los autores Ming Dong y Frank Chen, sugieren desarrollar un modelo de red de inventarios, incorporando políticas de reposición para la red integrada. Primero sugieren identificar las interdependencias que existen entre los actores y segundo desarrollar una red de modelos de reposición para analizar de desempeño de la red logística integrada con control de inventario en todos los sitios.

"La cadena productiva de cosméticos y aseo comprende la producción de detergentes y productos de aseo, jabones y cosméticos" [6]. Las funciones de una empresa de fabricación de cosméticos, pueden esquematizarse en una serie de actividades que están compuestos por procesos internos requeridos para entregar el producto terminado. La actividad primaria de la Empresa de Cosméticos S.A. de esta investigación es la fabricación de cosméticos, los recursos humanos y materiales, la recepción de la materia prima e insumos y los procesos internos están enfocados en productos de aseo, cuidado personal y cosméticos.

Para remarcar la gestión de inventarios basada en la demanda, es importante conocer la flexibilidad del suministro. Estudios e investigaciones recientes de la cadena de suministro proponen evaluar modelos de alineación de la estrategia de marketing, rendimiento de la cadena de suministro y el desempeño de la estructura organizacional. En la Empresa de Cosméticos S.A., uno de los principales objetivos es fortalecer las relaciones con los proveedores, cuenta con 230 proveedores, 80 proveedores proveen el $90 \%$ del suministro.

Algunas investigaciones presentadas a continuación han enfocado su estudio a la mejora en cuanto a la flexibilidad para el cambio de la demanda dentro de la cadena de abastecimiento.

Los investigadores Kenneth W. Green Jr., Dwayne Whitten y R. Anthony Inman en el 2012, demostraron la relación entre el éxito de las cadenas de abastecimiento y la alineación de las estrategias de marketing, los hallazgos preliminares entre 117 empresas, indican que las empresas exitosas están enfocadas al consumidor y tienen mayor alineación de las estrategias de marketing entre los socios de 
toda la cadena de abastecimiento, logrando mayor rendimiento y mejor desempeño organizacional [8].

Wantao Yu, Ramakrishnan Ramanathan y Prithwiraj Nath en 2014, investigaron la relación entre la capacidad de comercialización en la cadena de abastecimiento, la capacidad de las operaciones y los resultados financieros, demostrando que la capacidad de comercialización es considerada como una de las más importantes ventajas competitivas, ya que ayuda a fortalecer los lazos en las relaciones con los consumidores, mejora la comunicación para las operaciones internas y por lo tanto mejora el rendimiento financiero [9].

En un estudio no muy reciente pero pertinente para la presente investigación, Amelia S. Carr y John N. Pearson en 1999, mostraron la relación entre la función estratégica de compras y su influencia en los sistemas de evaluación de los proveedores, con el desempeño financiero de la empresa, las empresas que tienen una función de compras estratégicas son más proclives a implementar un sistema de evaluación de proveedores buscando relaciones a largo plazo como clave para tener un impacto positivo en los resultados financieros de la empresa [10].

En una encuesta realizada en el 2014 a 230 proveedores de la Empresa de Cosméticos S.A., se intenta validar la alineación de las estrategias de mercadeo, la capacidad de comercialización y la evaluación de los indicadores para fortalecer las relaciones de la cadena de abastecimiento a largo plazo, el resultado encontrado demuestra desconocimiento de las estrategias de marketing entre las empresas de la misma cadena de abastecimiento, no se evidencia el interés común por la mejora en el desempeño financiero y no se muestran estrategias claras para mejoras futuras.

Los miembros de la cadena de suministro han encontrado que es relevante el intercambio de información para mejorar la respuesta del abastecimiento, estudios realizados en dos empresas de manufactureras muestran el impacto al implementar un modelo analítico jerárquico, donde se clasifica la información disponible y cual información puede contribuir a mejorar la precisión de los pronósticos [11].

La gestión de proveedores directos estudiada por Brammer, sugiere el desarrollo de proveedores basado en mejorar las relaciones a largo plazo [12], clasificando la gestión de los proveedores en dos: La Evaluación y la Colaboración [13], la investigación hace alusión ampliada al paradigma de la colaboración, más allá de las operaciones centradas de la cadena de suministro.

La evaluación permite ver el rendimiento de los proveedores y su nivel de cumplimiento a la empresa, donde las empresas pueden aplicar criterios definidos para seleccionar proveedores capaces y reducir el riesgo que estos proveedores no cumplan los requerimientos de la empresa [14], los criterios y los estándares de evaluación se han convertido en una preocupación importante para las áreas de compras y gestión de abastecimiento, además de contar con una base de suministro cada vez más global. Mediante la realización de la compra responsable y la gestión de proveedores se puede evitar el riesgo de daño a la confiabilidad de la empresa [15].

\subsection{Estado del arte}

La modelación de las cadenas de abastecimiento tuvo su origen desde 1961. Forrester, considerado el padre de la dinámica de sistemas investigó como la modelación de los sistemas puede dar mayor confiabilidad a la toma de decisiones en las empresas [16].

Para demostrar el impacto de los inventarios en la cadena de abastecimiento, John David Sterman en 1989 creó un juego llamado "El juego de la cerveza", ampliamente conocido académicamente y usado en muchas empresas, para entender la dinámica que deben tener los inventarios ante la variabilidad de la demanda [17].

Continuando su estudio en el año 2000, el mismo autor publicó el libro Business Dynamics, donde muestra los beneficios de los modelos de simulación para el mundo complejo, reforzando así el riesgo en la toma de decisiones debido al uso de los modelos de simulación para el control de los inventarios [18].

La investigación de operaciones en la cadena de abastecimiento, se enfoca en optimizar y simular los posibles escenarios en la cadena de abastecimiento, para mejorar la toma de decisiones empresariales, una definición encontrada es " $\mathrm{La}$ investigación de operaciones es un planteamiento científico para la toma de decisiones, que busca como diseñar y operar mejor un sistema, normalmente bajo condiciones que requieran la asignación de recursos escasos" [19].

En la actualidad S\&OP, Sales and Operations Planning, "son una ciencia y un arte" [20], hoy, Supply Chain Council (SCC) and American Production and Inventory Control Society (APICS), están unidos como una organización, donde su principal función, es lograr cadenas de abastecimiento eficientes de acuerdo a la exigente globalización actual, diseñan y desarrollan metodologías aplicadas a lograr cadenas de abastecimiento exitosas. Estas organizaciones tienen las últimas tecnologías y metodologías de gestión aplicadas y validadas en las cadenas de abastecimiento, donde será una fuente importante de investigación para el presente proyecto, en los conceptos de pronósticos, reposición de inventarios y gestión de proveedores a los que hace referencia la presente investigación.

En la Empresa de Cosméticos S.A., se está comenzando con un plan estratégico para fortalecer las 
relaciones con los proveedores y así mejorar la flexibilidad requerida en el mercado como ventaja competitiva decisiva. Hasta el momento no se había hecho ninguna gestión, por lo tanto esta investigación es el punto de partida para la empresa.

La influencia en la compra, remarcada en la venta directa, está catalogada como un método de marketing eficaz, comparado con la costosa publicidad, las ventas directas pueden proporcionar productos $y$ servicios exclusivos para consumidores específicos. La referencia [21] proporciona en su investigación, información acerca del impacto del internet en la venta directa de cosméticos, los hábitos del consumidor, su estilo de vida y el comportamiento de compra. Los resultados de la investigación pueden ayudar a las empresas a comercializar sus productos por medio de la venta directa, abriendo mercados más amplios y obteniendo mayores ganancias.

La creciente evidencia de las ventas directas, indican que los comportamientos de los consumidores y los vendedores son factores claves del éxito para mejorar en el rendimiento de las ventas [22], dado que los consumidores y las ventas adaptadas al comportamiento del consumidor, afectan las acciones organizacionales, un siguiente paso en la investigación fue explorar cómo las organizaciones inducen los comportamientos de las personas de ventas, sin embargo, pocos estudios han sido examinados dentro de las organizaciones [23]. Investigaciones sobre la construcción de relaciones de quienes practican la venta directa, definen como retener las relaciones con los clientes [24], otros estudios hacen referencia a como los sistemas para el control de las ventas, son una herramienta de gestión importante para lograr los objetivos organizacionales deseados [25].

Las empresas se enfrentan cada vez más a la presión de los accionistas para mantener la rentabilidad de sus cadenas de suministro, lo cual hace necesario mejorar la gestión logística en su cadena de abastecimiento, además de asumir la responsabilidad de sus proveedores que garanticen los insumos y materiales requeridos, cualquiera de las partes de la cadena de suministros que no cumplan con los estándares de la empresa puede potencialmente dañar la confiabilidad que el cliente requiere [26].

\section{METODOLOGÍA}

La metodología de la presente investigación comienza con la identificación del problema, las posibles causas y las relaciones con los efectos encontrados, provee un camino para guiar la investigación, proporcionando las principales variables a estudiar con el objeto de construir un modelo apropiado para la gestión del abastecimiento en la Empresa de Cosméticos S.A.

La metodología de la presente investigación comienza con la identificación del problema, las posibles causas y las relaciones con los efectos encontrados, provee un camino para guiar la investigación, proporcionando las principales variables a estudiar con el objeto de construir un modelo apropiado para la gestión del abastecimiento en la Empresa de Cosméticos S.A.

La metodología a seguir, para el desarrollo del presente artículo, es un compendio de un proyecto de investigación específico para la empresa de cosméticos de venta directa, pero que puede ser útil para empresas similares del sector cosmético.

Identifica y desarrolla un modelo de gestión de abastecimiento requerido para reponer las necesidades de productos cosméticos para venta directa. La metodología está orientada a lograr el objetivo propuesto de "Proponer un modelo de gestión para suministro de materiales e insumos de acuerdo a los requerimientos de la demanda" (ver Tabla 1).

Dentro de la metodología en el paso cinco, se usan los simuladores como herramientas para analizar diferentes escenarios, analizar los impactos y disminuir el riesgo de implementación (ver Tabla 1).

Tabla 1. Pasos metodológicos para el desarrollo de la investigación.

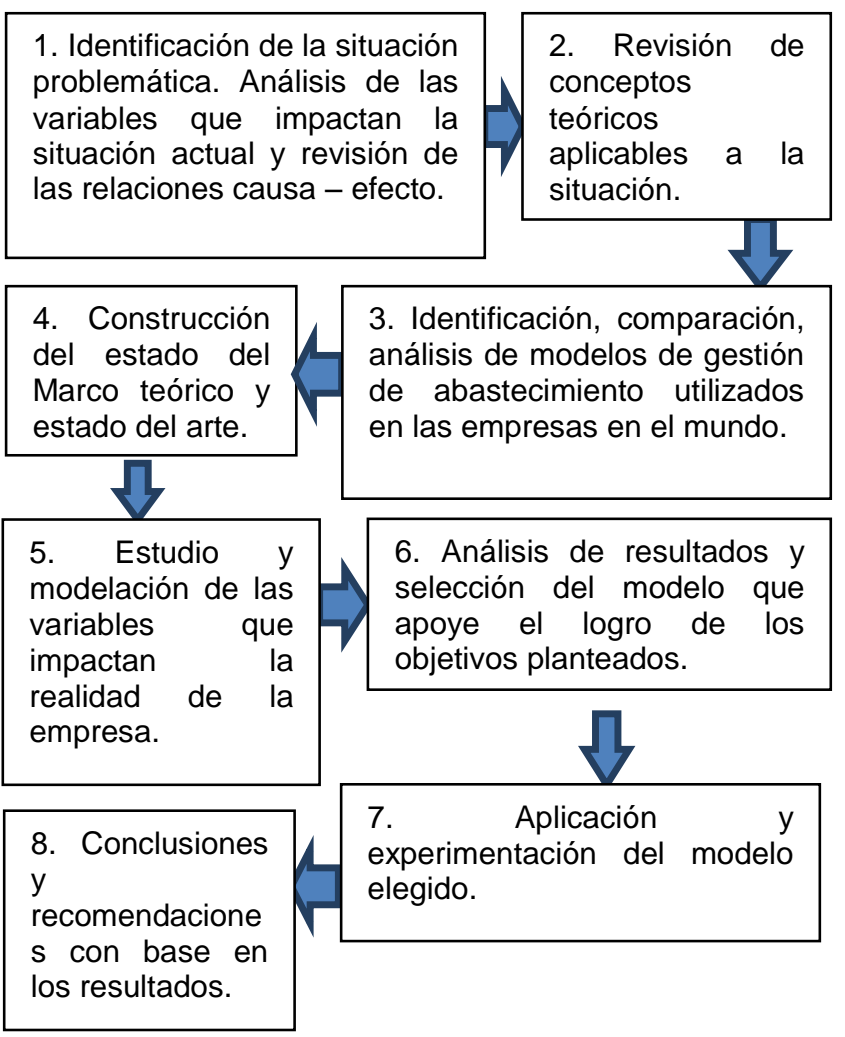

Fuente: elaboración propia 
Las herramientas para modelación de sistemas dinámicos se han usado en los últimos años para estudiar la forma de sincronizar acciones, dar propuestas ante diferentes escenarios y dar flexibilidad a las cadenas de abastecimiento [18].

En esta investigación, se ha usado el software Powersim, para simular la cantidad de inventario sometido a una demanda altamente variable y a diferentes escenarios, con el objeto de validar el impacto de variables específicas en la situación actual de la empresa, y encontrar mejoras que ayuden en la formulación de un modelo de abastecimiento que asegure la disponibilidad.

Los siguientes son los pasos para la elaboración del modelo de simulación. Dicha guía es elaborada con base en la simulación de sistemas dinámicos y los tutoriales del software para simulación de inventarios del Powersim Studio Academic [27].

Tabla 2. Pasos para modelar simulación.

1. Identificación del problema

2. Análisis de las relaciones causaefecto

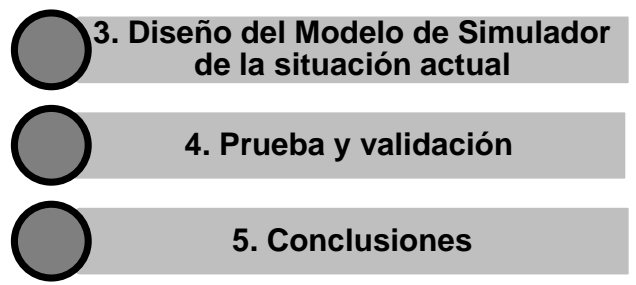

Fuente: elaboración propia

\subsection{Descripción de la situación problemática de la Empresa de Cosméticos S.A.}

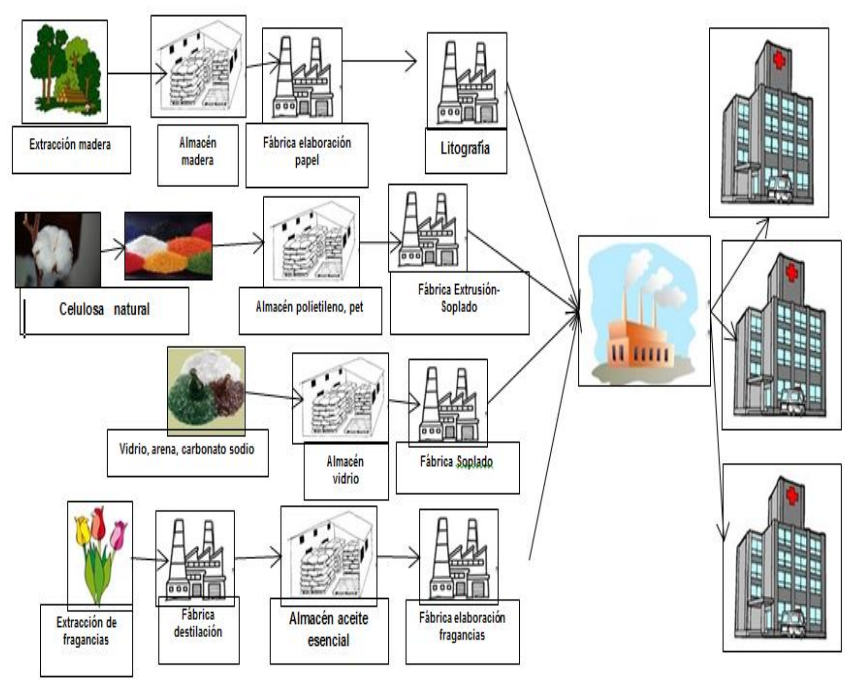

Fig. 1. Cadena de Abastecimiento de la Empresa de Cosméticos S.A. Fuente: elaboración propia.
La empresa en la que se ejecutó la presente investigación se caracteriza por ser una empresa de producción de cosméticos, que llega al consumidor final por medio del sector venta directa, con ventas promedio de $\$ 1.100$ millones de pesos al mes.

La cadena de abastecimiento está compuesta de 230 proveedores, los cuales abastecen cerca de 6000 SKUS, Su cadena de abastecimiento se encuentra descrita en la Fig. 1.

La Fig. 1 muestra el flujo del producto y de la información de la Empresa de Cosméticos S.A, base necesaria para cumplir el objetivo principal de esta investigación que es definir herramientas y estrategias de abastecimiento para el balanceo de los inventarios frente a la demanda. O desarrollar un modelo de gestión de abastecimiento para balancear los inventarios con el consumo

\subsubsection{Identificación y priorización de materiales según su valor de compra}

El valor de compra para cada material es diferente. Existen diferentes tipos de clasificación de los materiales, según la clasificación $A B C$, se clasifican los SKUS con relación al costo de compra de los materiales [3].

Según el especialista en inventarios, Carlos Julio Vidal [3], "Para definir la clasificación de los ítems, se escoge un porcentaje de mayor a menor, de acuerdo con el orden de utilización", en nuestro caso, los SKUS están elegidos por el orden del valor de compra. Usualmente los ítems clase A que van del 10\% al 20\% de los SKUS, representan entre el $60 \%$ y $80 \%$ del valor de las compras, clase B, están entre un $20 \%$ y $40 \%$ y representan entre el $15 \%$ y el $20 \%$ del valor de las compras y los ítems $\mathrm{C}$, son los más numerosos y casi nunca sobrepasan al $10 \%$ del valor total de las compras (ver Tabla 3). 
Tabla 3. Clasificación de los SKUS en la Empresa de Cosméticos S.A., según el valor de la compra.

\begin{tabular}{|c|c|c|c|c|c|c|}
\hline $\begin{array}{c}\text { Clasi } \\
\text { ficac } \\
\text { ión }\end{array}$ & $\begin{array}{c}\# \\
\text { SKUS }\end{array}$ & $\begin{array}{c}\% \text { de } \\
\text { SKUS }\end{array}$ & $\begin{array}{c}\text { Demanda } \\
\text { Total Uds } \\
\text { pedidas en } \\
\text { el 2013 }\end{array}$ & $\begin{array}{c}\text { Valor } \\
\text { compra } \\
\text { (suma } \\
\text { compras } \\
\text { año 2013) }\end{array}$ & $\begin{array}{c}\text { Compr } \\
\text { as } \\
\text { (valor } \\
\text { compr } \\
\text { a/total } \\
\text { compr } \\
\text { as) }\end{array}$ & $\begin{array}{c}\text { Promedio } \\
\text { Uds } \\
\text { (Demand } \\
\text { a total } \\
\text { uds/\# } \\
\text { SKUS) }\end{array}$ \\
\hline A & 33 & $2 \%$ & 7.091 .628 & 2.588 .719 .934 & $59,6 \%$ & 214.898 \\
\hline B & 51 & $3 \%$ & 5.110 .410 & $\$ 880.779 .436$ & $20.3 \%$ & 100.204 \\
\hline C & 1955 & $96 \%$ & 14.350 .857 & $\$ 871.557 .334$ & $20.1 \%$ & 7.341 \\
\hline Total & 2039 & & & & & \\
\hline
\end{tabular}

Fuente: elaboración propia información 2013.

La siguiente tabla muestra que el $96 \%$ de los SKU de la empresa son de poco volumen de compra, representan el $20 \%$ del total de las compras, lo que hace más difícil conocer cuánto inventario se debe mantener.

Una de las políticas organizacionales es preferir mantener inventario en materiales e insumos que en producto terminado, dada la alta variabilidad del mercado de venta directa. En los últimos 7 años, la empresa ha visto incrementado su inventario de manera considerable, la información del año 2014 se encuentra hasta junio (ver Tabla 4).

Tabla 4. Inventario últimos 7 años Empresa de Cosméticos S.A.

\begin{tabular}{|c|c|c|c|c|c|c|}
\hline & 2014 & $\%$ & Vr. & 2013 & $\%$ & Vr. \\
\hline MATERIA PRIMA & 670,477 & $\begin{array}{l}16 \\
\% \\
\end{array}$ & $4 \%$ & 700,300 & $\begin{array}{l}17 \\
\% \\
\end{array}$ & $\begin{array}{l}15 \\
\% \\
\end{array}$ \\
\hline $\begin{array}{l}\text { PRODUCTO } \\
\text { TERMINADO }\end{array}$ & 944,808 & $\begin{array}{r}23 \\
\%\end{array}$ & $4 \%$ & 983,088 & $\begin{array}{r}24 \\
\%\end{array}$ & $\begin{array}{l}3 \\
\%\end{array}$ \\
\hline $\begin{array}{l}\text { PRODUCTOS EN } \\
\text { PROCESO }\end{array}$ & 220,000 & $\begin{array}{r}5 \\
\% \\
\end{array}$ & $\begin{array}{r}22 \\
\% \\
\end{array}$ & 283,189 & $\begin{array}{r}7 \\
\% \\
\end{array}$ & $\begin{array}{l}12 \\
\% \\
\end{array}$ \\
\hline $\begin{array}{l}\text { MATERIAL DE } \\
\text { EMPAQUE }\end{array}$ & $2,938,278$ & $\begin{array}{r}70 \\
\% \\
\end{array}$ & $\begin{array}{r}24 \\
\% \\
\end{array}$ & $\begin{array}{l}2,364,13 \\
2\end{array}$ & $\begin{array}{r}57 \\
\% \\
\end{array}$ & $\begin{array}{r}7 \\
\% \\
\end{array}$ \\
\hline $\begin{array}{l}\text { PROVISION } \\
\text { INVENTARIOS }\end{array}$ & $\begin{array}{l}- \\
156,594\end{array}$ & $\begin{array}{r}4 \\
\%\end{array}$ & $2 \%$ & $\begin{array}{l}- \\
152,832\end{array}$ & 4 & $\begin{array}{r}0 \\
\%\end{array}$ \\
\hline TOTAL & $4,616,969$ & & & $\begin{array}{l}4,177,87 \\
7\end{array}$ & & \\
\hline CRECIMIENTO & $11 \%$ & & & $-5 \%$ & & \\
\hline $\begin{array}{l}\text { ROTACION INV. } \\
\text { (días) }\end{array}$ & 144 & & & 142 & & \\
\hline & 2012 & $\%$ & Var. & \multicolumn{3}{|c|}{2011} \\
\hline MATERIA PRIMA & 606,937 & $14 \%$ & $15 \%$ & \multicolumn{3}{|c|}{527,078} \\
\hline
\end{tabular}

\begin{tabular}{|c|c|c|c|c|c|c|}
\hline & 2014 & $\%$ & Vr. & 2013 & $\%$ & Vr. \\
\hline $\begin{array}{l}\text { PRODUCTO } \\
\text { TERMINADO }\end{array}$ & $1,009,094$ & $23 \%$ & $85 \%$ & \multicolumn{3}{|c|}{544,682} \\
\hline $\begin{array}{l}\text { PRODUCTOS EN } \\
\text { PROCESO }\end{array}$ & 252,495 & $6 \%$ & $23 \%$ & \multicolumn{3}{|c|}{329,347} \\
\hline $\begin{array}{l}\text { MATERIAL DE } \\
\text { EMPAQUE }\end{array}$ & $2,542,838$ & $58 \%$ & $2 \%$ & \multicolumn{3}{|c|}{$2,486,187$} \\
\hline $\begin{array}{l}\text { PROVISION } \\
\text { INVENTARIOS }\end{array}$ & 14,651 & $0 \%$ & $0 \%$ & \multicolumn{3}{|c|}{56,719} \\
\hline TOTAL & $4,396,713$ & & $15 \%$ & \multicolumn{3}{|c|}{$3,830,575$} \\
\hline CRECIMIENTO & $15 \%$ & & & & & $9 \%$ \\
\hline $\begin{array}{l}\text { ROTACION INV. } \\
\text { (días) }\end{array}$ & 126 & & & & 141 & \\
\hline
\end{tabular}

Fuente: adaptada de información Empresa de Cosméticos S.A. Junio de 2014.

En el 2014, el material de empaque representa un $70 \%$ del valor total de los inventarios, una cifra que ha mantenido similar desde el 2008. El exceso de inventarios genera un conflicto, los inventarios le ayudan a la empresa a proteger sus ventas, pero si hay altos inventarios se pone en peligro sus costos y su liquidez.

El material de empaque para el sector cosmético representa confianza, imagen, estilo y calidad, la presentación del producto es de alto impacto en las ventas, adicionalmente en el material de empaque va la información legal de cada producto exigido como el INVIMA. Debido a esto existe en la Empresa de Cosméticos S.A., gran variedad de material de empaque, se tiene 4300 materiales de empaque diferentes.

\subsubsection{Costos totales inherentes a los inventarios}

Tabla 5. Resumen costos totales inherentes a los inventarios.

\begin{tabular}{|l|c|}
\hline Costos mensuales & Valor en pesos (anual) \\
\hline $\begin{array}{l}\text { Costos de } \\
\text { inventarios } \\
\text { obsoletos }\end{array}$ & $\$ 898842.446$ \\
\hline $\begin{array}{l}\text { Costos de } \\
\text { inventarios activos }\end{array}$ & $\$ 4.234 .890 .000$ \\
\hline $\begin{array}{l}\text { Costos logísticos de } \\
\text { administración de } \\
\text { inventarios }\end{array}$ & $\$ 459.315 .000$ \\
\hline $\begin{array}{l}\text { Costos logísticos de } \\
\text { los inventarios }\end{array}$ & $\$ 5.593 .047 .446$ \\
\hline
\end{tabular}

Fuente: elaboración propia

De la tabla 5 , se observa que los costos inherentes a los inventarios para la empresa se encuentran principalmente: costos de inventarios obsoletos, costos de inventarios activos, costos logísticos de administración de inventarios, costos logísticos de los inventarios. 


\subsubsection{Costos logísticos de administración de inventarios}

Los costos de administración de inventarios a final del año 2013, tienen incluidos el valor de arrendamiento de bodegas y personal inherente a la logística de inventarios, estos fueron de $\$ 459.315 .000$.

\subsubsection{Duración en días de los inventarios activos.}

La duración de los inventarios muestra un problema grave, algunas referencias de material de empaque están con sobre inventario. Por ejemplo, en 2014, del estuche HX-129B se tienen 17.741 unidades en existencia, con un consumo de 3.37 unidades día, la duración de dicho material, serán 5269 días, aproximadamente 14,4 años, cifra que es alarmante.

\subsubsection{Nivel de servicio y agotados}

El nivel de servicio, medido como el cumplimiento en fecha y unidades de acuerdo al compromiso del cliente (Numero de pedidos cumplidos/total de pedidos), es uno de los indicadores más importantes de la empresa, todos los procesos están orientados cada día a cumplir los requerimientos del cliente, es un indicador monitoreado constantemente en el proceso, debido a la alta exigencia en el proceso de venta directa, la alta competencia y a los altos costos por no entregar lo pedido. Por estar en un sector ampliamente competido, es vital para sus ventas tener disponibilidad de producto terminado cuando la vendedora lo requiera.

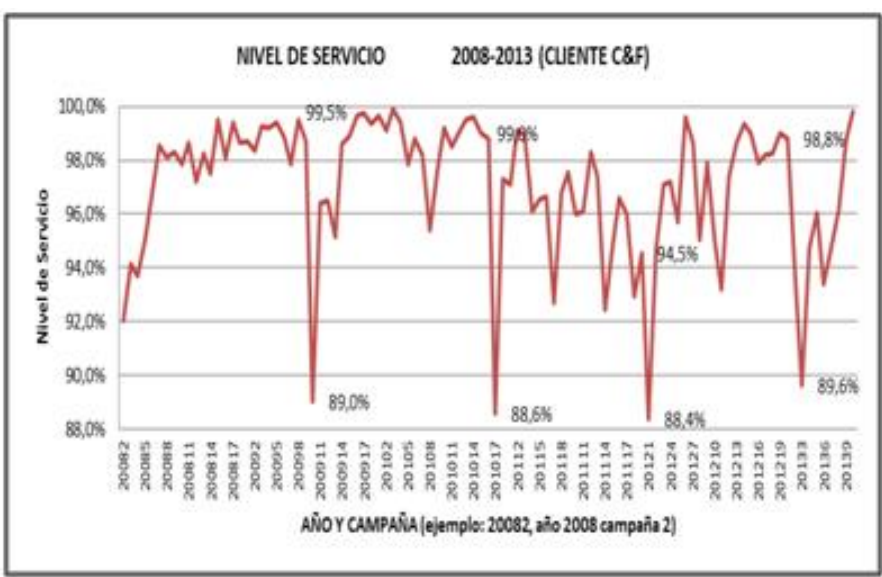

Fig. 2. Nivel de servicio. Fuente: elaboración propia.

Según los resultados obtenidos de la Fig. 2, un nivel de servicio de aproximadamente el $88 \%$, puede representar perdidas en ventas por campaña por valor promedio de $\$ 200$ millones de pesos.

\subsubsection{Agotados}

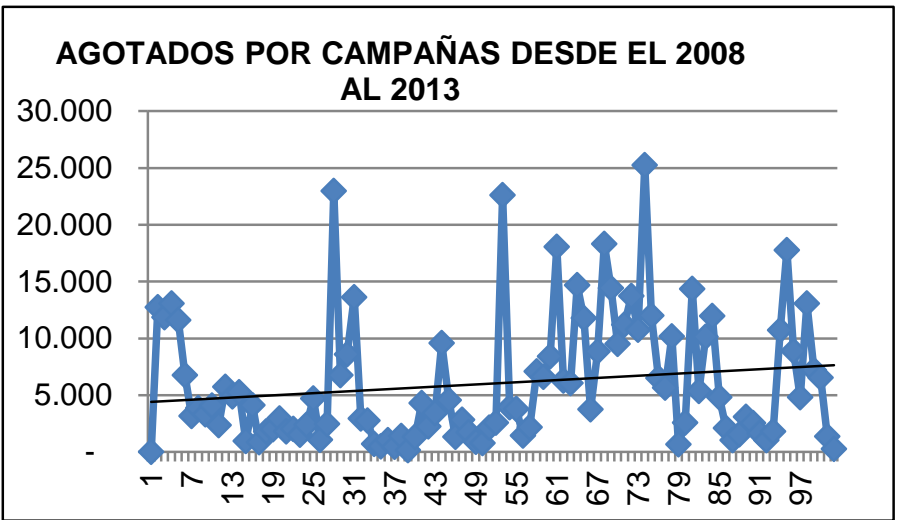

Fig. 3. Agotados

Fuente: elaboración propia. Adaptada de: información Empresa de Cosméticos S.A. Octubre 2013.

En la Fig. 3, analizando la información de la Empresa, se encuentra una tendencia ascendente. El nivel de servicio bajo, genera agotados.

\subsubsection{Asertividad en los pronósticos de la venta directa}

El sector de venta directa, usa pronósticos econométricos para predecir la demanda, se hizo una evaluación de la asertividad de dichos pronósticos, (dividiendo el pedido real/pedido estimado) mostrando que solo el $32 \%$ de los productos solicitados, tienen una asertividad entre el $80 \%$ y $120 \%$, el $41 \%$ de los SKUS pedidos, tienen una asertividad en el pronóstico entre $0 \%$ y $80 \%$ y el $39 \%$ restante tienen una asertividad en el pronóstico entre 120\% y 1000\% (ver Fig. 4).

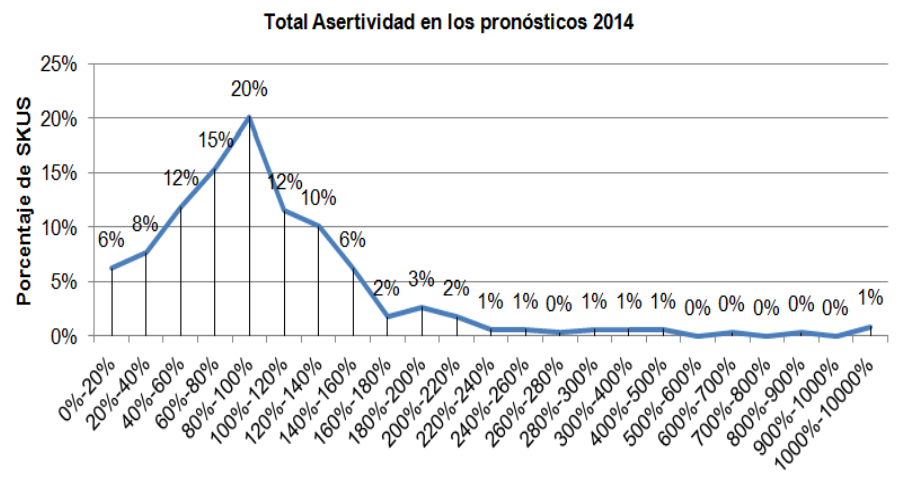

Porcentaje de asertividad en el pronóstico

Fig. 4. Asertividad en los pronósticos 2014.

Fuente: elaboración propia. Adaptada de: información Empresa de Cosméticos S.A. 2014. 


\subsubsection{Relación entre lo presupuestado, pedido y facturado}

El $90 \%$ de las ventas se hacen a clientes de ventas por catálogo, dicho sector presenta una alta variación entre lo presupuestado y lo pedido por campaña. En la Tabla 6, podemos visualizar las unidades presupuestadas, pedidas y facturadas por año desde el 2008 hasta el 2013.

Tabla 6. Relación entre lo presupuestado, pedido y facturado

\begin{tabular}{|l|l|l|l|}
\hline Año & $\begin{array}{l}\text { Presupuest } \\
\text { ado }\end{array}$ & Pedido & $\begin{array}{l}\text { Facturad } \\
\text { o }\end{array}$ \\
\hline $\mathbf{2 0 0 8}$ & 202.940 & 199.009 & 193.342 \\
\hline $\mathbf{2 0 0 9}$ & 252.284 & 214.446 & 209.966 \\
\hline $\mathbf{2 0 1 0}$ & 250.331 & 186.313 & 182.641 \\
\hline $\mathbf{2 0 1 1}$ & 259.167 & 237.296 & 228.014 \\
\hline $\mathbf{2 0 1 2}$ & 233.736 & 187.724 & 181.146 \\
\hline $\mathbf{2 0 1 3}$ & & & \\
\hline
\end{tabular}

Fuente: elaboración propia. Adaptada de: información de Empresa de Cosméticos S.A., datos hasta campaña 10 en el año 2013.

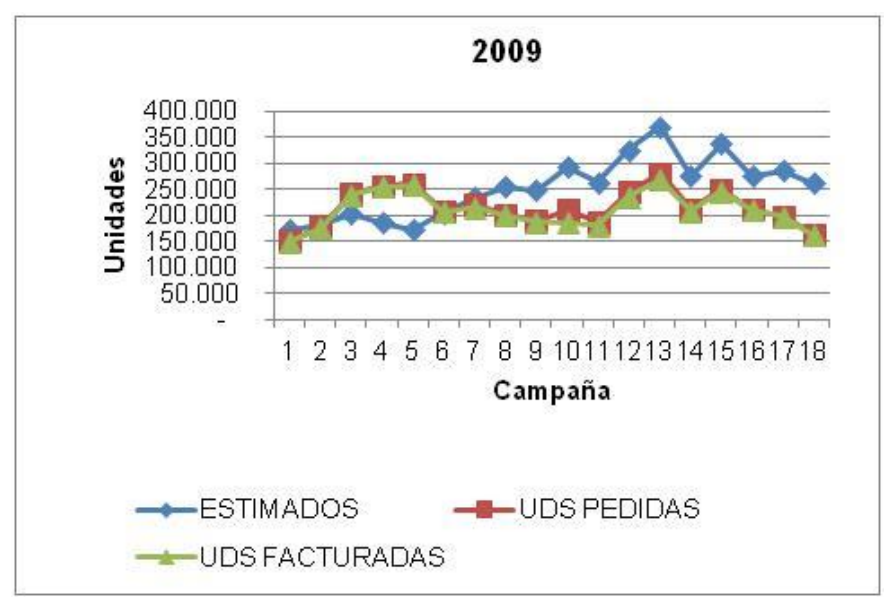

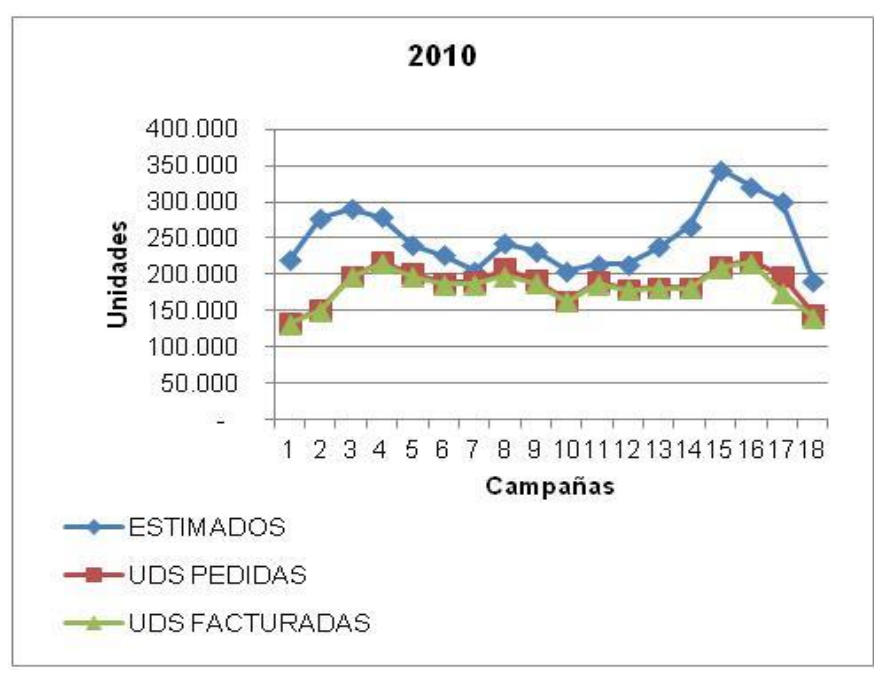
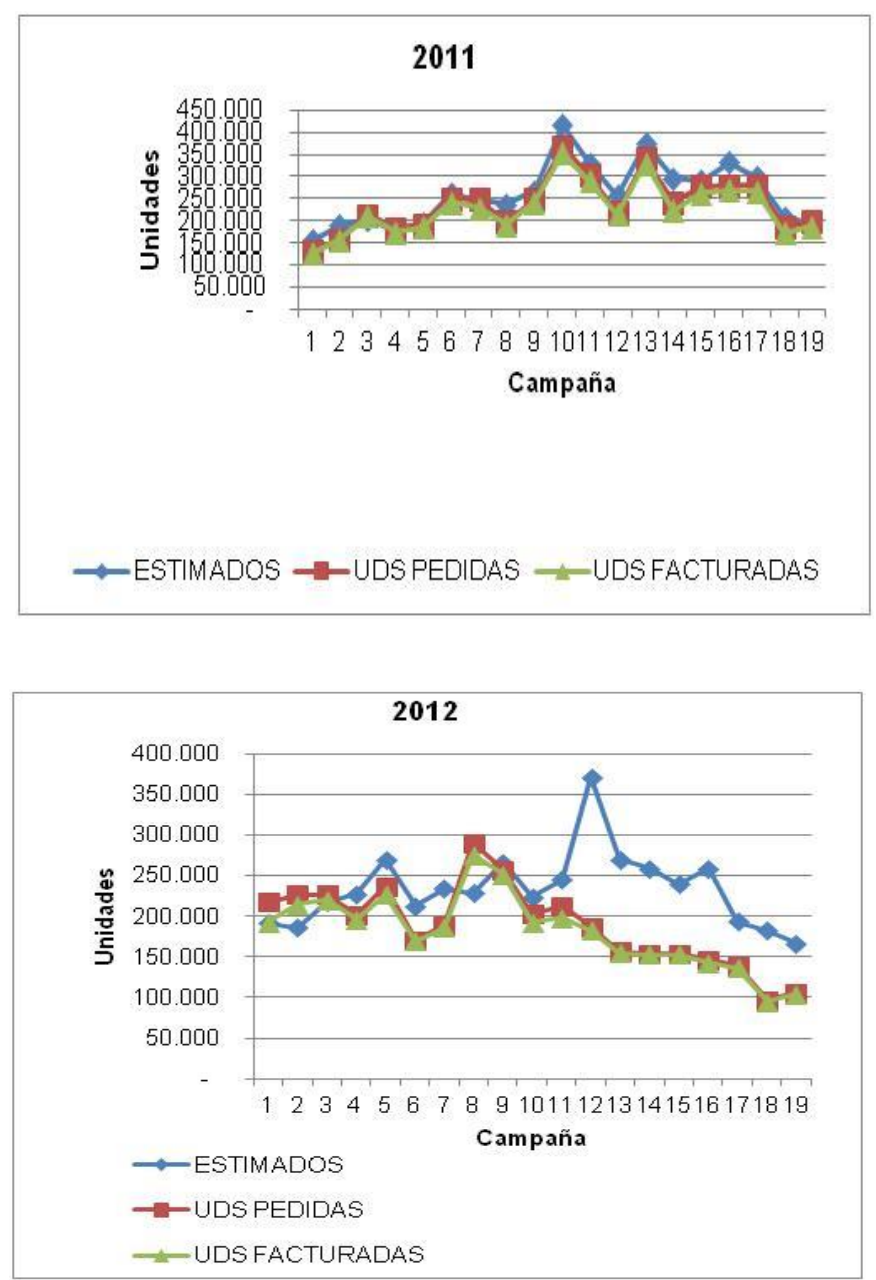


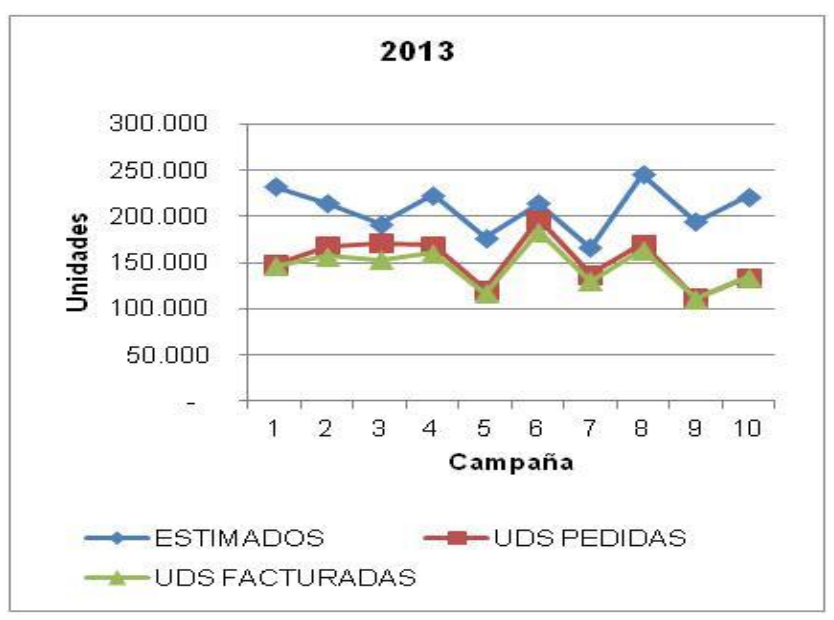

Fig. 5. Gráficas con estimados, unidades pedidas y unidades facturadas de 2009 a 2013.

Fuente: elaboración propia con datos de Empresa de Cosméticos S.A.

De la Fig. 5, datos recopilados desde 2009 hasta 2013 puede verse que financieramente el exceso de inventario, genera poca liquidez en algunos periodos de la empresa para su funcionamiento normal y la sostenibilidad en el tiempo.

\subsection{Caracterización de la venta directa}

La venta directa está constituida por actividades de venta multinivel, se define como toda actividad de mercadeo, de promoción o de ventas, donde se incorporan personas naturales con el fin de vender bienes y servicios, quienes obtienen compensaciones, pagos $u$ otros beneficios de cualquier índole, por la venta de bienes y servicios a través de las personas involucradas [28].

Las empresas que comercializan sus productos por medio de la venta directa, fundamenta sus estrategias de ventas en cada catálogo, donde cada uno representa un escenario diferente, diferente focalización del mercado objetivo y diferentes ofertas [29].

Cada vendedor puede usar diferentes estilos de ventas, entre los cuales se pueden identificar los siguientes: Catálogo como herramienta para comercializar, venta puerta a puerta, asistir y promocionar "Puerta a Puerta"; modalidad manejada principalmente por Avon en sus inicios, además existen las reuniones demostrativas que es el estilo de venta particular más importante utilizado por Tupperware [8].

Cada empresa coordina el lanzamiento por "Campañas", lo cual consiste en ofrecer los productos de los folletos o catálogos de ventas durante periodos determinados, generalmente cortos, con una duración no mayor a 21 días y con 18 o 19 campañas por año.

Se planea la campaña por medio de proyecciones de unidades a vender, llamadas "tendencias" [30].

La corta duración de las campañas para la venta directa, hace más exigente los cortos tiempos, tanto en la fabricación como en el suministro de los materiales requeridos, obligando así a toda la cadena de abastecimiento a ser agiles, flexibles y a tener una gestión de inventarios que garantice disponibilidad, pero al mismo tiempo bajos inventarios.

\subsection{Simulación del flujo del proceso en la cadena de abastecimiento de la empresa.}

Los simuladores son herramientas administrativas, que le permiten al empresario simular diferentes situaciones o escenarios, que puedan impactar el resultado final esperado.

En la Empresa de Cosméticos S.A. se formuló el proceso de su cadena de abastecimiento, simulando las variables como: variación en la demanda, Inventarios de materiales y producto terminado, el tiempo de reposición de los materiales desde el proveedor y el tamaño de lote de compra.

El simulador de la Fig. 6 socializado con la dirección de la empresa, refleja el movimiento real de los productos en la cadena de abastecimiento de la empresa, razón por la cual, se hace importante comenzar a analizar el impacto de las variables diseñadas en el simulador, en el resultado final, para poder establecer políticas para el control de los inventarios. 


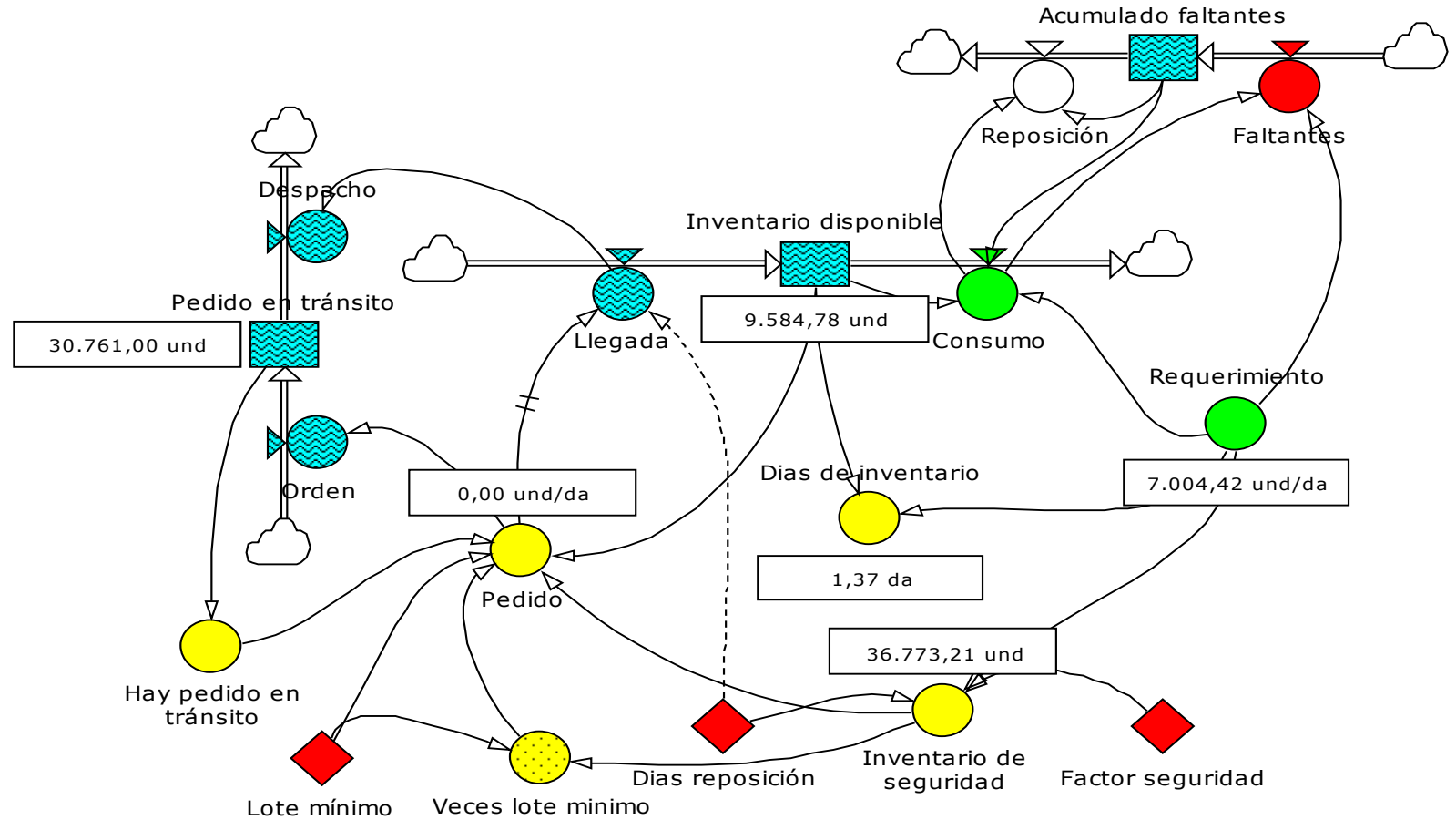

Fig. 6. Cadena de abastecimiento de la Empresa de Cosméticos S.A. Fuente: elaboración propia.

\subsubsection{Funciones usadas en la simulación}

La tabla 7 presenta las funciones usadas en la simulación de la cadena de abastecimiento de la Empresa de Cosméticos S.A:

Tabla 7. Funciones principales de la simulación.

\begin{tabular}{|c|c|c|c|}
\hline Name & Unit & Uetinition & Value 5 \\
\hline \multirow{6}{*}{$\begin{array}{l}\square \text { Inventario disponible } \\
\square \text { 生 Consumo.out } \\
\Rightarrow \text { Llegada.in } \\
\Rightarrow \text { Faltantes }\end{array}$} & \multirow[t]{3}{*}{ und } & $50000\langle\langle$ und $\rangle\rangle$ & \multirow[t]{3}{*}{$9.584,783928482$ und } \\
\hline & & Consumo & \\
\hline & & Llegada & \\
\hline & und/da & Requerimiento-Consumo & 766,8695840563 und/da \\
\hline & und/da & RANDOM $(3500<\langle$ und $/$ da $>>; 8000<\langle$ und $/$ da $>>)$ & $7,004,421550372$ und/da \\
\hline & und/da & $\begin{array}{l}\text { MIN('Inventario } \\
\text { disponible't } 1<\langle 1 / \text { da } \gg>\text {; Requerimiento+'Acumulado } \\
\text { faltantes' } 1<\langle 1 / \text { da } \gg>\text { ) }\end{array}$ & $6.237,551966316$ und/da \\
\hline Dias reposición & da & $5\langle\langle d a\rangle\rangle$ & 5 da \\
\hline Inventario de seguridad & \multirow[t]{2}{*}{ und } & 'Dias reposición'^'Factor seguridad'*Requerimiento & $36.773,21313945$ und \\
\hline Veces lote minimo & & 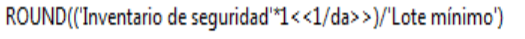 & 36.773 \\
\hline Pedido en tránsito & \multirow[t]{3}{*}{ und } & $0<\langle$ und $\gg\rangle$ & \multirow[t]{3}{*}{30.761 und } \\
\hline 䚾 Despacho.out & & Despacho & \\
\hline$\stackrel{\ddagger}{\ddagger}$ Orden.in & & Orden & \\
\hline Dias de inventario & \multirow[t]{2}{*}{ da } & 'Inventario disponible'/Requerimiento & $1,368390502992 \mathrm{da}$ \\
\hline Factor seguridad & & 1,05 & 1,05 \\
\hline Lote mínimo & und/da & $1\langle\langle$ und $/$ da $\rangle\rangle$ & 1 und $/ d a$ \\
\hline Llegada & und/da & DELAYPPL(Pedido;'Dias reposición';0<<und/da〉>) & 0 und $/ d a$ \\
\hline Pedido & und/da & $\begin{array}{l}\text { IF('Inventario disponible' }\langle=\text { 'Inventario de seguridad';'Veces lote } \\
\text { minimo'*'Lote mínimo'; } 0<\langle\text { und } / \text { da } \gg>)^{*} \text { Hay pedido en tránsito' }\end{array}$ & 0 und $/$ da \\
\hline Despacho & und/da & Llegada & 0 und $/ d a$ \\
\hline Orden & \multirow[t]{2}{*}{ und/da } & Pedido & 0 und $/ d a$ \\
\hline Hay pedido en tránsito & & IF('Pedido en tránsito' $>0<<$ und $>>; 0 ; 1)$ & 0 \\
\hline 2. Reposición & und/da & MIN('Acumulado faltantes't $1\langle\langle 1 /$ da $\gg>$; Consumo) & $-766,8695840563$ und/da \\
\hline \multirow{3}{*}{ 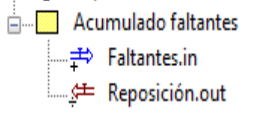 } & \multirow[t]{3}{*}{ und } & $0\langle\langle$ und $\gg\rangle$ & \multirow[t]{3}{*}{$-766,8695840563$ und } \\
\hline & & Faltantes & \\
\hline & & Reposición & \\
\hline
\end{tabular}

Fuente: elaboración propia 


\subsubsection{Explicación de las funciones usadas en la simulación}

\section{Inventario disponible}

El inventario disponible son las unidades disponibles para la demanda al comienzo de la simulación. Este se disminuye con el consumo y se alimenta con la llegada de inventario del proveedor.

\section{Faltantes}

Los faltantes resultan de la resta entre el requerimiento y lo que se despachó, en este caso lo llamamos consumo.

\section{Requerimiento}

El requerimiento, es lo que solicita la demanda, está diseñado con la función RANDOM, genera una serie de números aleatorios que se distribuyen de acuerdo con una distribución uniforme, con un valor mínimo y un máximo.

\section{Consumo}

El Consumo, es lo que se puede despachar al cliente, está definido con las unidades mínimas entre el inventario disponible y el requerimiento.

\section{Días de reposición}

Los días de reposición es una constante que define el proveedor.

\section{Inventario de seguridad}

El inventario de seguridad, está calculado por el requerimiento, multiplicado por los días de reposición de los proveedores y un factor de seguridad para proteger la variación.

\section{Llegada de inventario}

Las llegada de inventarios desde el proveedor está calculada con la función DELAYPPL función de retardo del tiempo de reposición.

\section{ANÁLISIS DE LA INFORMACIÓN. RESULTADOS Y DISCUSIÓN}

\subsection{Análisis del impacto de las variables en la simulación}

Se usó el simulador de inventarios para la empresa, en un SKU con demanda intermitente (SKU que no tiene oferta en todas las campañas del año), se analizará dentro del tiempo de la campaña 18.

La Tabla 8, muestra las actividades dentro de una campaña de venta directa, donde se puede ver (si no se tiene material disponible) que solo se dispone de 11 días para realizar el proceso desde el proveedor hasta entregar el producto terminado a la venta directa. 
Tabla 8. Actividades en una campaña de venta directa

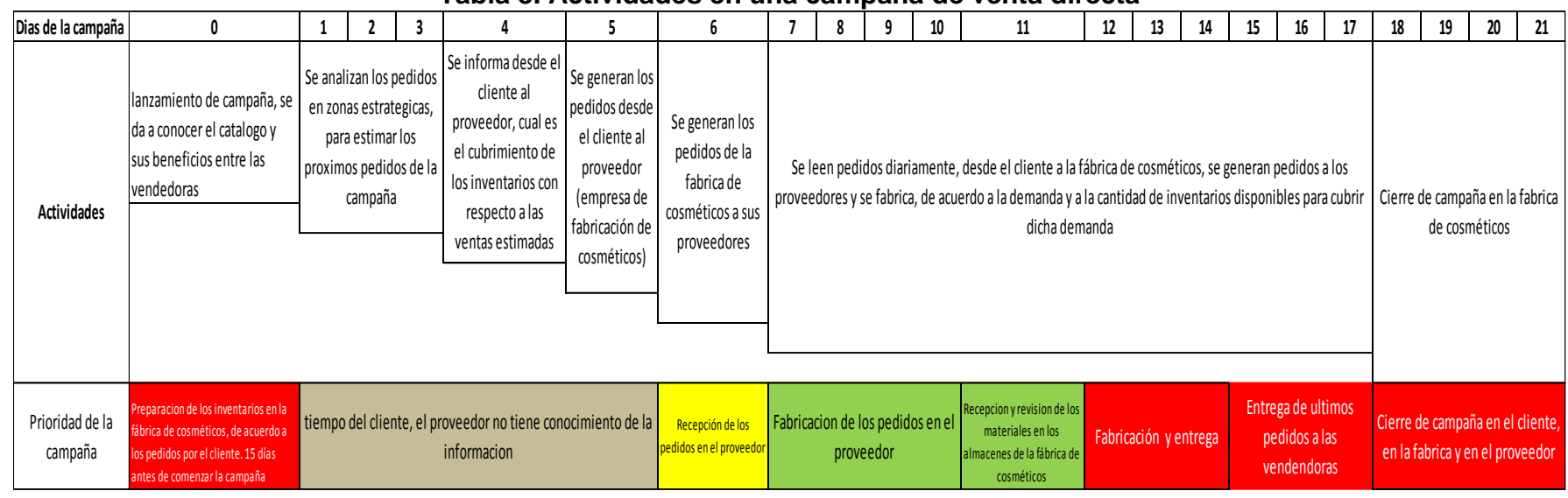

Fuente: elaboración propia.

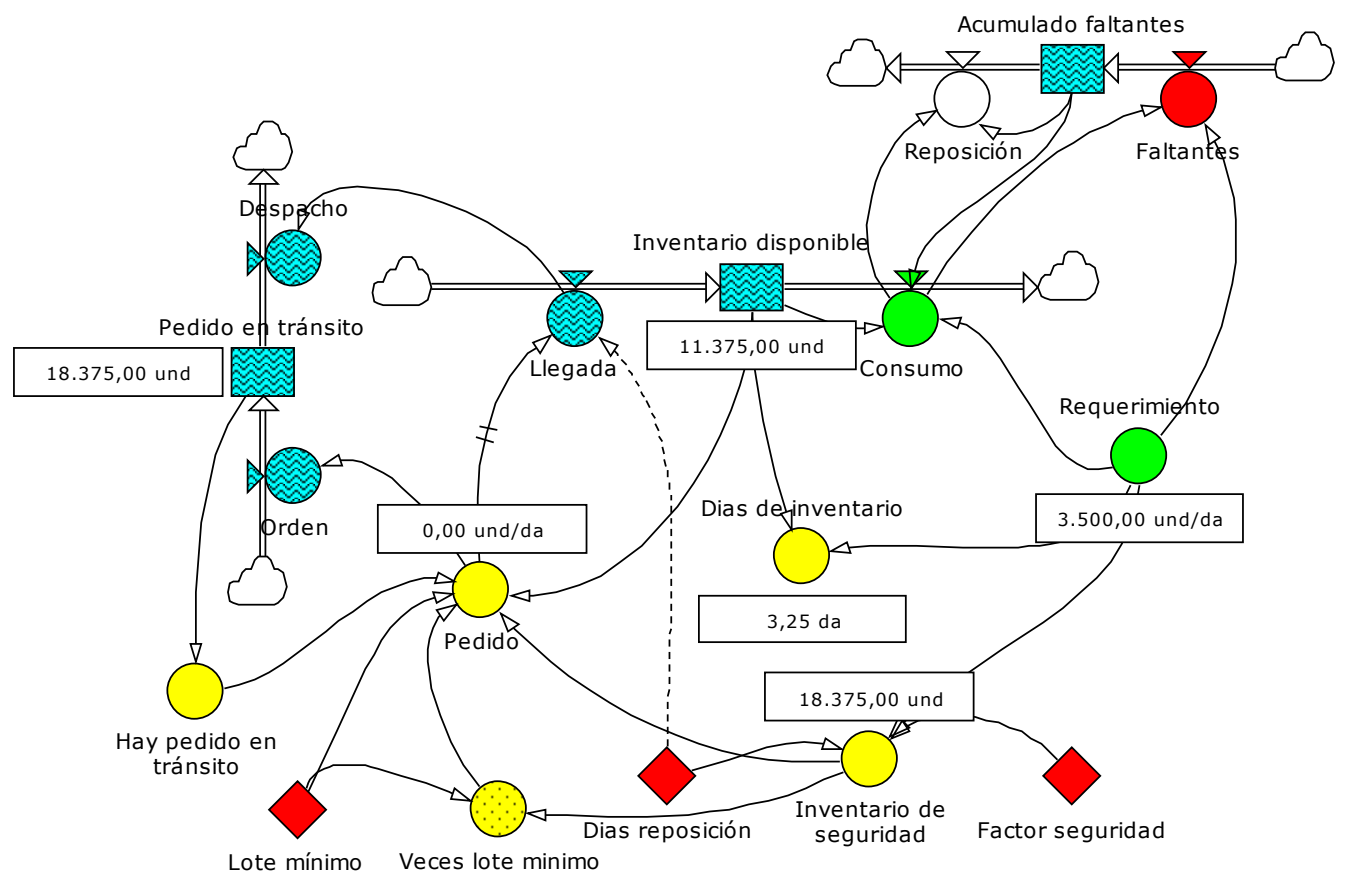

Fig. 7. Simulación de la realidad de las campañas de venta directa Fuente: elaboración propia

Cuando la demanda es constante, se puede visualizar claramente cuando pedir los inventarios y cuanto inventario pedir de acuerdo a dichos requerimientos (ver Fig. 8).

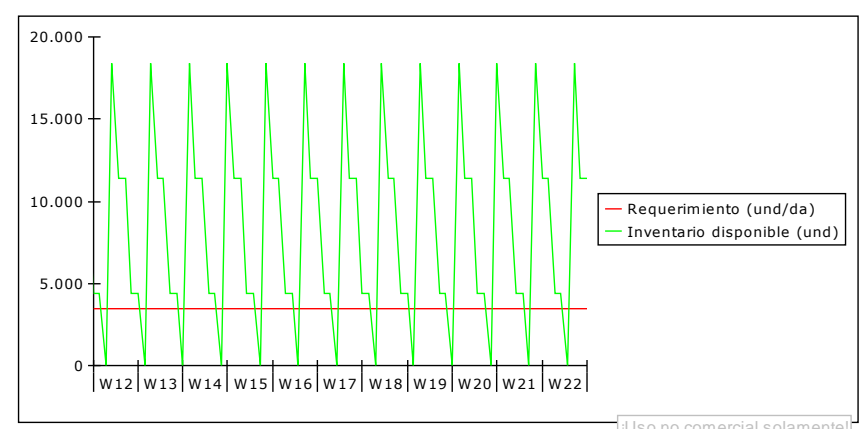

Fig 8. Requerimiento versus inventario disponible con demanda constante. Fuente: elaboración propia
En esta situación, sería muy sencillo saber que tenemos un suministro de materiales en exceso para la demanda constante.

Como la realidad es que la demanda varía, se simuló la variabilidad y se analizó lo sucedido con el suministro y los agotados de productos al mercado

Tomaremos una variación de la demanda, usando la función RANDOM (3500 《 unds/día >>; $8000 \ll$ unds / día >>), para tomar un numero aleatorio entre 3500 y 8000 unds. La Fig.9 muestra la variación de la demanda y el exceso de inventarios que se tienen para cubrirla. 


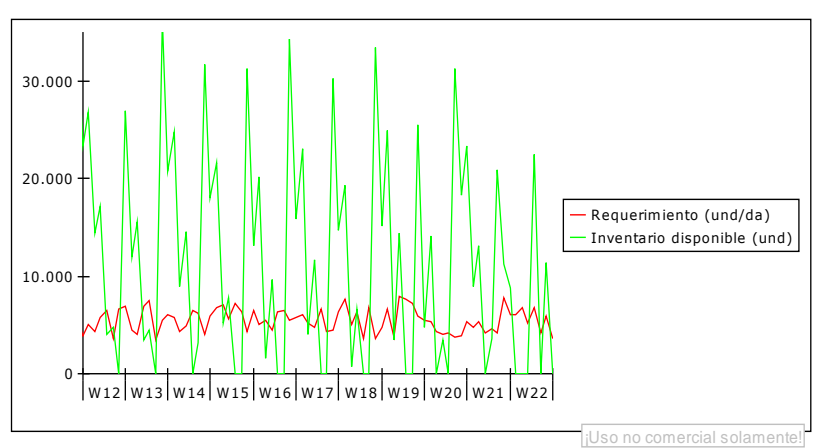

Fig 9. Requerimiento versus inventario disponible con demanda variable entre 3500 y 8000 unds Fuente: elaboración propia

Tabla 9. Tiempo comparado con el inventario disponible, el inventario de seguridad y el pedido en tránsito.

\begin{tabular}{|c|c|c|c|}
\hline \multicolumn{4}{|c|}{ (und) } \\
\hline Tiempo & Inventario disponible & Inventario de seguridad & Pedido en tránsito \\
\hline \multicolumn{4}{|l|}{27 de dic de 2014} \\
\hline 04 de ene de 2015 & $35.375,46$ & $28.678,31$ & 0,00 \\
\hline 11 de ene de 2015 & 0,00 & $30.761,40$ & $28.560,00$ \\
\hline 18 de ene de 2015 & 0,00 & $19.611,31$ & $36.836,00$ \\
\hline 25 de ene de 2015 & $40.653,00$ & $28.170,64$ & 0,00 \\
\hline 02 de feb de 2015 & $37.069,00$ & $29.531,90$ & 0,00 \\
\hline 09 de feb de 2015 & 0,00 & $39.533,94$ & $31.059,00$ \\
\hline 16 de feb de 2015 & $33.113,00$ & $23.826,25$ & 0,00 \\
\hline 23 de feb de 2015 & $20.104,00$ & $32.382,22$ & 0,00 \\
\hline 30 de feb de 2015 & $13.418,05$ & $35.573,64$ & 0,00 \\
\hline 07 de mar de 2015 & $15.408,77$ & $36.798,05$ & 0,00 \\
\hline 14 de mar de 2015 & $23.417,99$ & $20.337,78$ & 0,00 \\
\hline 21 de mar de 2015 & $26.998,00$ & $36.350,11$ & 0,00 \\
\hline 28 de mar de 2015 & $20.776,60$ & $31.647,44$ & 0,00 \\
\hline 05 de abr de 2015 & $18.097,98$ & $31.189,31$ & 0,00 \\
\hline 12 de abr de 2015 & $13.200,64$ & $34.235,55$ & 0,00 \\
\hline 19 de abr de 2015 & $15.943,25$ & $30.189,69$ & 0,00 \\
\hline 26 de abr de 2015 & $14.684,13$ & $33.398,63$ & 0,00 \\
\hline 03 de may de 2015 & $15.101,30$ & $25.567,79$ & 0,00 \\
\hline 10 de may de 2015 & $4.772,22$ & $29.074,19$ & $31.224,00$ \\
\hline 17 de may de 2015 & $23.419,02$ & $28.568,37$ & $20.842,00$ \\
\hline 24 de may de 2015 & $8.774,35$ & $32.070,94$ & $22.455,00$ \\
\hline 01 de jun de 2015 & 0,00 & $18.930,05$ & $35.611,00$ \\
\hline & & & \\
\hline & & & \\
\hline
\end{tabular}

Fuente: elaboración propia

Al revisar los datos se puede ver que hay días donde el inventario disponible llega a cero, poniendo en peligro el cumplimiento de los pedidos que haga el mercado, además se puede visualizar un cálculo del inventario objetivo con unidades suficientes para cubrir la demanda, pero un inventario en tránsito interrumpido, lo que puede poner en peligro el suministro continuo de materiales.

Dando respuesta a la pregunta ¿Cómo proteger el nivel de servicio sin aumentar los inventarios?, debemos de analizar los métodos existentes para el control de inventarios. Los inventarios son usados para responder rápidamente al mercado que no está dispuesto a esperar y que se puede convertir en una oportunidad para la competencia.

Existen dos formas para la gestión del desajuste de los inventarios entre la oferta y la demanda [31], la primera es la planificación de las necesidades que surge de la proyección de los pronósticos, cuando la demanda es conocida, los inventarios requeridos encajan perfectamente a la demanda, la segunda forma de gestión de inventarios, cuando la demanda es incierta y no conocida, el control estadístico del inventario se da por el método de extraer, donde los niveles de inventario para cada punto se deciden independientemente de los demás puntos de la cadena de abastecimiento y por el método de empujar, donde se asignan inventarios de acuerdo al tiempo del sistema de distribución, las necesidades previstas, la demanda real, el espacio o cualquier otro criterio [31].

En la simulación mostrada en la Fig. 10, se disminuye el tiempo de reposición, para validar la cantidad de inventarios y la protección que tenemos de la demanda.

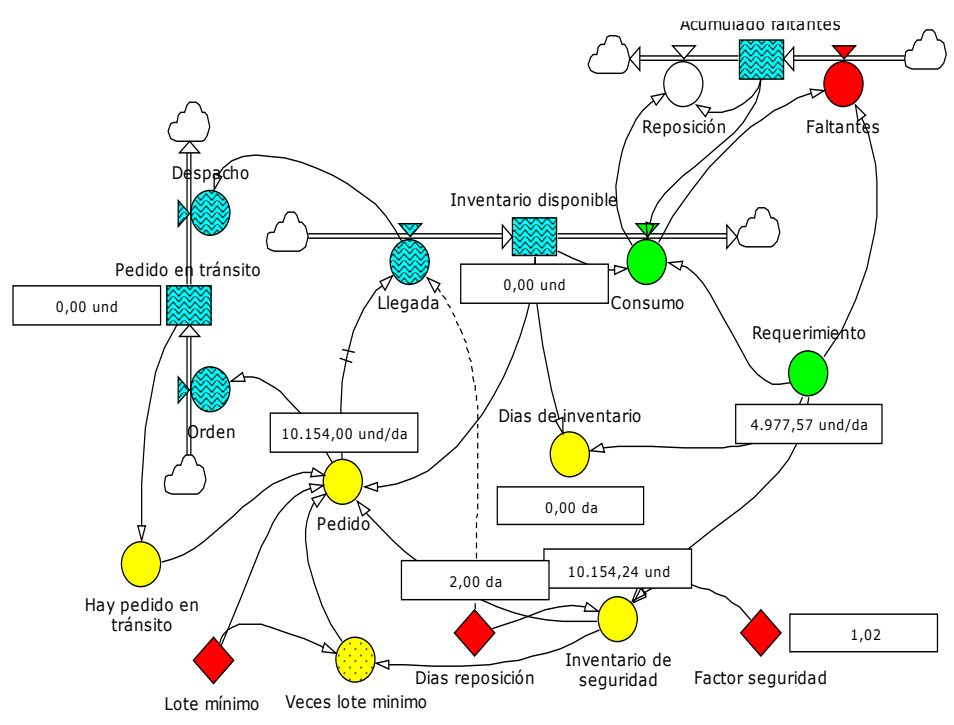

Fig. 10. Requerimiento versus inventario disponible Fuente: elaboración propia

La cantidad de inventario disminuye (ver Fig. 11).

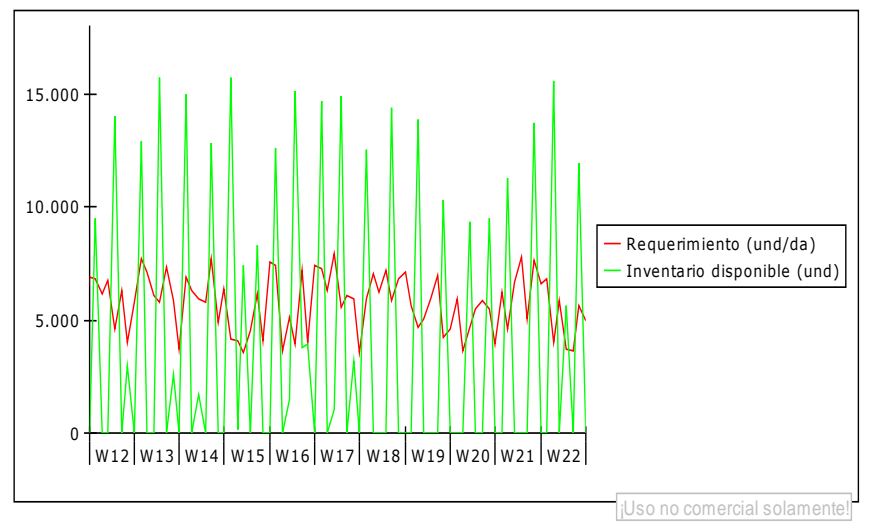

Fig 11. Requerimiento versus inventario disponible con disminución del tiempo de reposición y disminución de la protección del inventario. Fuente: elaboración propia 
Tabla 10. Tiempo comparado con el inventario disponible, el inventario de seguridad, el pedido en tránsito y acumulado faltante.

\begin{tabular}{|c|c|c|c|c|}
\hline \multicolumn{5}{|c|}{ (und) } \\
\hline Tiempo & Inventario disponible & Inventario de seguridad & Pedido en tránsito & Acumulado faltantes \\
\hline \multicolumn{5}{|l|}{27 de dic de 2014} \\
\hline 04 de ene de 2015 & $32.686,78$ & $15.150,67$ & 0,00 & 0,00 \\
\hline 11 de ene de 2015 & 0,00 & $13.037,87$ & $10.777,00$ & $8.330,30$ \\
\hline 18 de ene de 2015 & 0,00 & $7.798,96$ & $13.237,00$ & $14.012,46$ \\
\hline 25 de ene de 2015 & 0,00 & $10.622,57$ & $13.715,00$ & $6.722,85$ \\
\hline 02 de feb de 2015 & 0,00 & $12.974,95$ & $16.250,00$ & $-4.099,36$ \\
\hline 09 de feb de 2015 & 0,00 & $11.009,70$ & $12.432,00$ & $-3.374,71$ \\
\hline 16 de feb de 2015 & 0,00 & $10.143,03$ & 0,00 & $-5.271,57$ \\
\hline 23 de feb de 2015 & 0,00 & $8.201,70$ & $11.823,00$ & $-1.624,42$ \\
\hline 30 de feb de 2015 & 0,00 & $9.066,93$ & $15.637,00$ & $6.614,11$ \\
\hline 07 de mar de 2015 & $3.536,40$ & $11.322,72$ & $8.010,00$ & $7.462,93$ \\
\hline 14 de mar de 2015 & 0,00 & $14.096,81$ & $9.486,00$ & $6.167,32$ \\
\hline 21 de mar de 2015 & 0,00 & $11.794,90$ & $12.904,00$ & $7.319,31$ \\
\hline 28 de mar de 2015 & 0,00 & $7.626,37$ & $14.934,00$ & $10.549,05$ \\
\hline 05 de abr de 2015 & 0,00 & $13.029,34$ & $15.703,00$ & $4.924,55$ \\
\hline 12 de abr de 2015 & 0,00 & $15.407,01$ & $12.575,00$ & $4.098,91$ \\
\hline 19 de abr de 2015 & 0,00 & $15.113,26$ & $14.679,00$ & $3.460,79$ \\
\hline 26 de abr de 2015 & 0,00 & $7.321,65$ & $12.500,00$ & $8.908,37$ \\
\hline 03 de may de 2015 & 0,00 & $14.559,08$ & $13.883,00$ & $6.805,48$ \\
\hline 10 de may de 2015 & 0,00 & $9.378,89$ & 0,00 & $1.653,28$ \\
\hline 17 de may de 2015 & 0,00 & $7.986,54$ & $11.293,00$ & $-324,46$ \\
\hline 24 de may de 2015 & 0,00 & $13.527,41$ & $15.537,00$ & $-276,83$ \\
\hline 01 de jun de 2015 & 0,00 & $10.154,24$ & 0,00 & $-6.330,27$ \\
\hline & & & & \\
\hline & & & & \\
\hline
\end{tabular}

Fuente: elaboración propia
Dicho cambio pone en riesgo todo el sistema, en la tabla anterior podemos visualizar la cantidad de inventario disponible, la mayoría de las veces esta en 0 , aumentando los faltantes o agotados.

Mantener información oportuna, del comportamiento de la demanda, puede ser una alternativa para mejorar la respuesta del abastecimiento

La comunicación con el proveedor es clave, informar como medida de control la cantidad de inventarios y el cubrimiento de la demanda, puede generar mejores decisiones, y proteger el nivel de servicio.

Se debería establecer control de inventarios en red, y gestión de amortiguadores, con el fin de mejorar la rotación de los inventarios en la cadena de abastecimiento.

La figura 13 muestra donde deben estar los amortiguadores de inventario, y como se deben priorizar las decisiones de acuerdo al movimiento de la demanda.

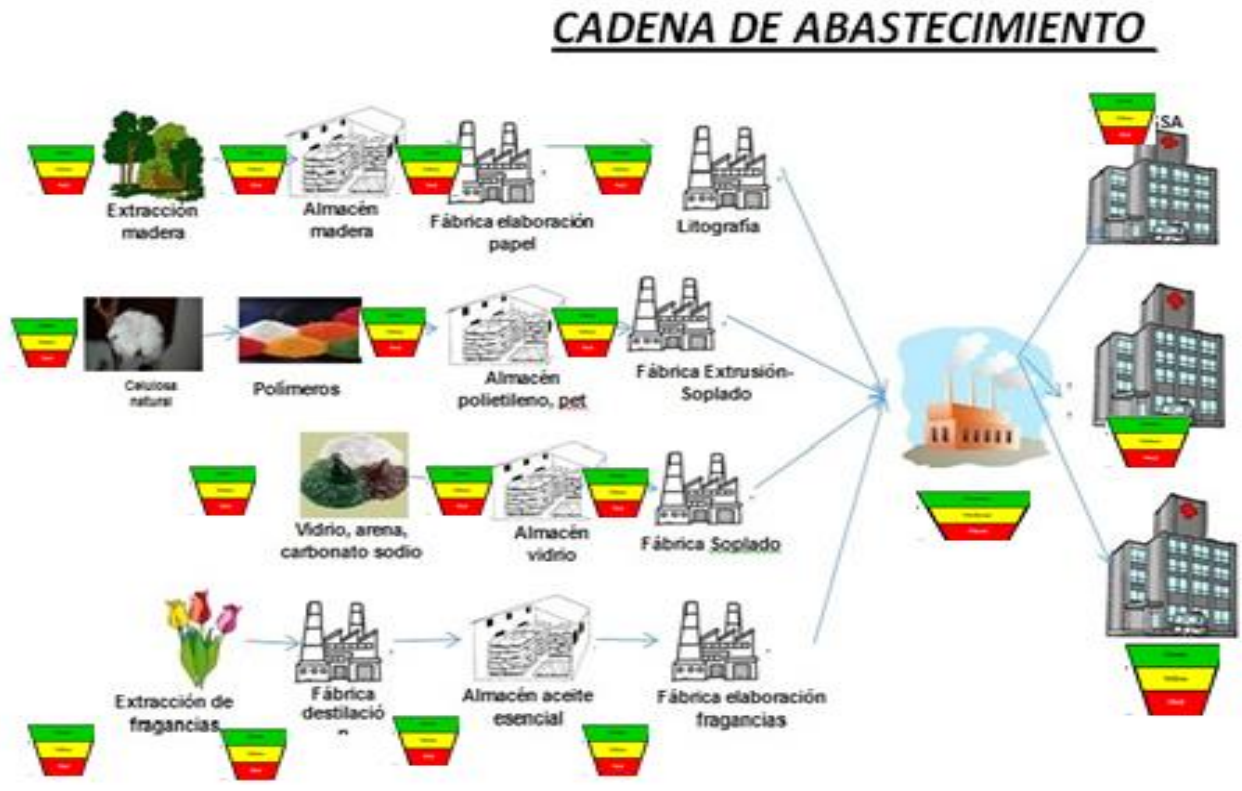

Fig. 13. Cadena de abastecimiento con amortiguadores de inventario Fuente: elaboración propia

La Fig. 13 muestra donde deben estar ubicados los amortiguadores de inventario. El amortiguador está identificado con tres colores dada la criticidad del inventario, según Goldratt en su TOC, la administración de los amortiguadores es una herramienta simple, se divide el inventario disponible sobre el inventario objetivo, multiplicado por 100, este porcentaje muestra el cubrimiento del inventario disponible con respecto a la política de inventario a mantener, consta de el color rojo, son los SKUS cuyo cubrimiento de inventario está entre $0 \%$ y $30 \%$, el color amarillo entre $31 \%$ y $66 \%$ de cubrimiento y el color verde entre el $67 \%$ y $100 \%$ de cubrimiento, estos colores ayuda a focalizar las acciones a emprender en cada caso. 


\section{RECOMENDACIONES}

Monitorear diariamente los inventarios en la cadena de suministro, requiere que las empresas involucradas estén interesadas en el reabastecimiento colaborativo, la participación de las empresas de la cadena de abastecimiento genera estrategias que permiten evitar las acciones reactivas [32]. La Planificación, Pronósticos y Reabastecimiento Colaborativo (CPFR) usa la información de los modelos de negocios colaborativos, los pronósticos y direccionamiento del flujo de productos e inventarios entre las partes involucradas en la cadena de abastecimiento.

Los pasos definidos por el CPFR y adaptados a las necesidades del presente proyecto son: 1. Establecer relaciones colaborativas, el comprador y el vendedor establecen lineamientos para dicha relación, se definen indicadores claves de la colaboración. 2. Se crea un plan de negocio conjunto de acuerdo con sus estrategias de mercadeo, en el cual se pueda compartir información para dicho fin. 3. Establecer estrategias de colaboración para responder a la sobredemanda y subdemanda del mercado de venta directa [33].

El modelo propuesto de abastecimiento con base en la demanda tiene cuatro etapas: (ver Tabla 11).

\section{Tabla 11. Modelo de abastecimiento con base en la} demanda

\begin{tabular}{|c|c|c|c|}
\hline \multicolumn{4}{|c|}{ MODELO DE ABASTECIMIENTO CON BASE EN LA DEMANDA } \\
\hline ETAPA 1 & ETAPA 2 & ETAPA 3 & ETAPA 4 \\
\hline $\begin{array}{l}\text { Selección, } \\
\text { evaluación y } \\
\text { desarrollo } \\
\text { proveedores }\end{array}$ & $\begin{array}{l}\text { Planeación del } \\
\text { requerimiento de } \\
\text { la demanda, para } \\
\text { skus } \\
\text { intermitentes. }\end{array}$ & $\begin{array}{l}\text { Análisis de SKU bajo el } \\
\text { método GARCH. } \\
\text { Reposición de inventarios } \\
\text { en red. (Uso de los } \\
\text { modelos de simulación) } \\
\text { Definición de indicadores }\end{array}$ & $\begin{array}{l}\text { Alianzas estratégicas } \\
\text { Indicadores } \\
\text { Volver a etapa 1: Mejoras } \\
\text { propuestas de la alianza } \\
\text { que genera nuevos } \\
\text { criterios para la selección, } \\
\text { evaluación y desarrollo de } \\
\text { proveedores. }\end{array}$ \\
\hline \multicolumn{2}{|c|}{ PLANEACIÓN } & \multicolumn{2}{|c|}{ EJECUCIÓN } \\
\hline
\end{tabular}

Fuente: elaboración propia

Según la tabla 7 , el modelo propuesto para cada campaña, debería ser de la siguiente forma:

Tabla 12. Modelo de abastecimiento con acciones a tomar para cada campaña.

\begin{tabular}{|l|l|}
\hline Día & \multicolumn{1}{c|}{ Acciones a tomar } \\
\hline 0 & $\begin{array}{l}\text { Apertura de campaña } \\
\text { Se debe establecer una política de cubrimiento de } \\
\text { inventarios de acuerdo al estimado (o pedido del } \\
\text { cliente) de la próxima campaña. }\end{array}$ \\
\hline 1 al 3 & $\begin{array}{l}\text { Estos días es el lanzamiento de la campaña no se } \\
\text { tiene ninguna información para tomar decisiones. }\end{array}$ \\
\hline
\end{tabular}

\begin{tabular}{|l|l|}
\hline \multicolumn{1}{|c|}{ Día } & \multicolumn{1}{|c|}{ Acciones a tomar } \\
\hline 4 al 6 & $\begin{array}{l}\text { Compartir información de los estimados leídos por } \\
\text { el cliente dentro de su campaña, las posibles } \\
\text { sobreventas y las subdemandas, tomar } \\
\text { decisiones de comprar o parar órdenes de compra } \\
\text { en toda la cadena de abastecimiento. }\end{array}$ \\
\hline 7 al 17 & $\begin{array}{l}\text { Monitorear diariamente el cubrimiento de los } \\
\text { inventarios, las cantidades a pedir solo deben } \\
\text { responder a los requerimientos de la demanda, } \\
\text { debe compartirse toda la información en la } \\
\text { cadena de abastecimiento, tanto la información de } \\
\text { cubrimientos de inventario, como los inventarios } \\
\text { disponibles en cada punto (Ver Fig.13). }\end{array}$ \\
\hline 18 al 21 & $\begin{array}{l}\text { Cierre de campaña } \\
\text { Se debe evaluar, los inventarios generados, la } \\
\text { forma de evacuarlos y los sobrecostos generados } \\
\text { por las sobreventas. }\end{array}$ \\
\hline
\end{tabular}

Fuente: elaboración propia

\section{CONCLUSIONES}

- Las diferentes simulaciones realizadas permiten validar cuales son las variables de mayor impacto en la cantidad de inventario, como lo son: \% de protección y el retardo en la entrega de los proveedores, a mayor \% protección y retardo, mayor es el inventario de materiales, la disminución de los inventarios debe estar soportada por la mejora en la velocidad de respuesta del proveedor al cliente, debido a una adecuada y oportuna información.

- Las simulaciones permiten evaluar diferentes alternativas, como la cantidad de inventario a mantener, para proteger la demanda cambiante. A mayor variación de la demanda mayor incertidumbre del proceso para determinar la cantidad de inventario a mantener, los modelos de reposición de inventario como VMI, Lean y TOC, dan un acercamiento apropiado a la solución de control de inventarios, enfocado a mejorar en el desempeño financiero entre las empresas de la cadena de abastecimiento.

- Las variabilidad de la demanda puede generar altos inventarios o agotados de los mismos, los cuales son costosos para toda la cadena, esto puede generar un gran impacto en la imagen del consumidor final.

- Según el SKU analizado en el simulador, el cual tiene demanda intermitente, que para el caso particular es el $90 \%$ de los productos. I mantener comunicación constante del comportamiento de los amortiguadores, daría información de los requerimientos que tiene toda la cadena de 
abastecimiento, con el fin de proteger la demanda. Se recomienda hacer simulaciones con diferentes SKUS que representen los diferentes comportamientos de la demanda real, analizar el comportamiento del inventario con demandas intermitentes, y analizar el impacto de los pedidos intermitentes en la realidad de los proveedores para saber gestionar la cadena de abastecimiento.

- La Empresa de Cosméticos S.A. presenta alta incertidumbre en cuanto al desconocimiento de la demanda del mercado o conocer su sus tendencias o comportamiento, dado que un $90 \%$ de los productos ofrecidos, tienen demandas intermitentes a lo largo de la oferta de mercadeo por venta directa. Esta situación no le permite predecir y controlar los inventarios a comprar, es importante realizar un análisis de redes de inventario sugeridas para la cadena de abastecimiento, donde las colas de inventario son asumidas como protección a la incertidumbre del mercado.

- El aporte del modelo propuesto debe ser aplicado para toda la cadena de abastecimiento y monitorear con indicadores. Los beneficios del modelo están reflejados en la alineación de los inventarios de sus proveedores con los cambios de la demanda, la mejora de la rotación de los inventarios y la mejora del nivel de servicio al mercado. Una red integrada para el control de inventario agrega las respuestas de inventario para esta empresa, en cuanto al compartir la información y comenzar a crear alianzas estratégicas con los proveedores.

\section{REFERENCIAS}

[1] Congreso de Colombia. (2004). Ley 590 de 2004. Bogotá D.C., Colombia: Congreso de Colombia.

[2] John E. Hanke, (2010). Pronósticos en los Negocios. México: Pearson Educación.

[3] C. J. Vidal, (2010). Fundamentos de Control y Gestión de Inventarios. Cali. Colombia: Universidad Del Valle.

[4] Mee-Shew Cheung; Matthew B. Myers and John T. Mentzer. (2010). Does relationship learning lead to relationship value? Journal of Operations Management 28, 472-487.

[5] Y. Yu; Z. Hong; L.L. Zhang; L. Liang and C. Chu, (March 1 of 2013). Optimal selection of retailers for a manufacturing vendor in a vendor managed inventory system. European Journal of Operational Research, 225(2), 273-284.
[6] DNP. (2011). Cosméticos y Aseo. Disponible en: http://www.dnp.gov.co/Programas/

[7] Ming Dong and F. Frank Chen. (2005). Performance modeling and analysis of integrated logistic chains. European Journal of Operational Research, 83-98.

[8] Kenneth W. Green Jr.; Dwayne Whitten and R. Anthony Inman. (2012). Aligning marketing strategies throughout the supply chain to enhance performance. Industrial Marketing Management, 1008-1018.

[9] Wantao Yu; Ramakrishnan Ramanathan and Prithwiraj Nath. (2014). The impacts of marketing and operations capabilities on financial performance in the UK retail sector. Industrial Marketing Management, 25-31.

[10] Amelia S. Carr, John N. Pearson. (1999). Strategically managed buyer-seller relationships and performance outcomes. Journal of Operations Management 17, 497-519.

[11] Usha Ramanathan. (2013). Aligning supply chain collaboration using Analytic Hierarchy Process. Omega, 431-440.

[12] S. H. Brammer, (2011). http://nbs.net/wpcontent/uploads/NBS-Systematic-Review-Supply-

Chains. Obtenido de http: //nbs.net/wpcontent/uploads/NBS-Systematic-Review-SupplyChains.

[13] S. Vachon, R. K. (2006). Extending green practices across the supply chain: the impact of upstream and downstream integration. Int. J. Oper. Prod. Manag.

[14] C. Reuter, K. F. (2010). Sustainable global supplier management: the role of dynamic capabilities in achieving competitive advantage. Journal Supply Chain Management, 45-63.

[15] K. Foerstl, C. R. (2010). Managing supplier sustainability risks in a dynamically changing environment-Sustainable supplier management in the chemical industry. Journal of Purchasing and Supply Management, 118-130.

[16] Jay Wright Forrester. (1961). Industrial Dynamics. Massachusetts Institute of Technology Press.

[17] John D. Sterman. (1989). Modeling managerial behavior: misperceptions of feedback in a dynamic decision making experiment. London Business School: McGraw-Hill Higher Education.

[18] John D. Sterman. (2000). Business Dynamics: Systems Thinking and Modeling for a Complex World. London Business School: McGraw-Hill Higher Education.

[19] W.L. Winston, (2005). Investigación de Operaciones: Aplicaciones y Algoritmos. México: Editorial Iberoamérica.

[20] Lyengar Charanyan and Gupta Sandeep. (2013). Eight Building Blocks for Successful S\&OP. Supply Chain Management Review, 10-17. 
[21] S.-H Liao; Y.-J Chen, (2011). Mining customer knowledge for direct selling and marketing. Expert Systems with Applications, Vol. 38 Issue 5, p60596069. $11 \mathrm{p}$.

[22] W.J. Verbeke, B. D. (2011). Drivers of sales performance: A contemporary meta-analysis. Have salespeople become knowledge brokers? Journal of the Academy of Marketing Science, 407-428.

[23] P. Guenzi, L. D. (2011). Organizational drivers of salespeople's customer orientation and selling orientation. Journal of Personal Selling and Sales Management, 269-285.

[24] C.H. Schwepker Jr., D. G. (2012). Sales quotas: Unintended consequences on trust in organization, customer-oriented selling, and sales performance. Journal of Marketing Theory and Practice, 437-452.

[25] C.F. Miao, K. E. (2013). The interactive effects of sales control systems on salesperson performance: A job demands - Resources perspective. Journal of the Academy of Marketing Science, 73-90.

[26] Jörg H. Grimm, J. S. (2014). Critical factors for subsupplier management: A sustainable food supply chains perspective. International Journal of Production Economics, Pages 159-173.

[27] Software Powersim Studio Academic. (2005). Powersim Software AS (C).

[28] Congreso de la República de Colombia. (2013). Ley Multinivel 1700 del 27 de Diciembre de 2013.

[29] Antonio José Boada; Rómulo Mayorca. (2011). Planificación de la demanda en empresas con estilo de venta directa. Revista de Investigación LaSallista, 128-230.

[30]F. Correa, (10 de Mayo de 2014). Modelo de Pronóstico usado en el sector de venta directa. (I. C. Arango, Entrevistador).

[31] Ronald H. Ballou. (2004). Logística: Administración de la cadena de suministro. Ciudad de México: Prentice Hall.

[32] Reuben E. Slone, J. Paul Dittman and John T. Mentzer. (2011). Transformando la cadena de suministro. Harvard Business, Profit Editorial.

[33] Soluciones SAP, Software Empresariales \& Aplicaciones TI. (2014). [en línea]: disponible en: www.sap.com. 